\title{
Strong Aversive Conditioning Triggers a Long-Lasting Generalized Aversion
}

\section{Raul Ramos ${ }^{1 \dagger}$, Chi-Hong Wu ${ }^{1 \dagger}$, Gina G. Turrigiano ${ }^{1 *}$}

$2{ }^{1}$ Department of Biology, Brandeis University, Waltham, MA 02453, USA

$3 \dagger$ These authors have contributed equally to this work and share first authorship

4 * Correspondence:

5 Gina Turrigiano

6 turrigiano@brandeis.edu

7 Keywords: Conditioned Taste Aversion, Learning, Memory, Generalization, Synaptic

8 Plasticity, Gustatory Cortex

\section{Abstract}

10 Generalization is an adaptive mnemonic process in which an animal can leverage past learning

11 experiences to navigate future scenarios, but overgeneralization is a hallmark feature of anxiety

12 disorders. Therefore, understanding the synaptic plasticity mechanisms that govern memory

13 generalization and its persistence is an important goal. Here, we demonstrate that strong CTA

14 conditioning results in a long-lasting generalized aversion that persists for at least two weeks. Using

15 brain slice electrophysiology and activity-dependent labeling of the conditioning-active neuronal

16 ensemble within the gustatory cortex, we find that strong CTA conditioning induces a long-lasting

17 increase in synaptic strengths that occurs uniformly across superficial and deep layers of GC.

18 Repeated exposure to salt, the generalized tastant, causes a rapid attenuation of the generalized

19 aversion that correlates with a reversal of the CTA-induced increases in synaptic strength. Unlike the

20 uniform strengthening that happens across layers, reversal of the generalized aversion results in a

21 more pronounced depression of synaptic strengths in superficial layers. Finally, the generalized

22 aversion and its reversal do not impact the acquisition and maintenance of the aversion to the

23 conditioned tastant (saccharin). The strong correlation between the generalized aversion and synaptic

24 strengthening, and the reversal of both in superficial layers by repeated salt exposure, strongly

25 suggests that the synaptic changes in superficial layers contribute to the formation and reversal of the

26 generalized aversion. In contrast, the persistence of synaptic strengthening in deep layers correlates 
with the persistence of CTA. Taken together, our data suggest that layer-specific synaptic plasticity mechanisms separately govern the persistence and generalization of CTA memory.

\section{Introduction}

30 The cellular and synaptic physiology underlying the formation, storage, and retrieval of memories

31 has been extensively studied (Kandel et al., 2013; Kandel, Dudai, \& Mayford, 2014; Josselyn \&

32 Tonegawa, 2020), but comparatively less research has focused on the synaptic plasticity mechanisms

33 that govern the generalization of memories (Asok, Kandel, \& Rayman, 2019). Generalization is an

34 adaptive memory process by which an animal is able to extend its learning, garnered from past

35 experiences, to future similar scenarios (Shepard, 1987; Asok, Kandel, \& Rayman, 2019). In this

36 way, generalization endows organisms with greater cognitive flexibility and makes them more savvy

37 survivors. However, generalization can also be maladaptive, and overgeneralization of aversive

38 memories has been implicated in post-traumatic stress disorder and anxiety disorders (Kheirbek et

39 al., 2012; Mahan \& Ressler, 2012; Dunsmoor \& Paz, 2015). Thus, expanding our understanding of

40 the synaptic plasticity mechanisms that shape memory generalization and its persistence is of great

41 importance.

42 Conditioned Taste Aversion (CTA) learning is an ethologically relevant form of associative learning

43 in which experience with a novel tastant (CS) is paired with $\mathrm{LiCl}$ induced gastric malaise (US) to

44 produce a learned aversion (Bures, Bermúdez-Rattoni, \& Yamamoto, 1998; Reilly \& Schachtman,

45 2008). Unlike other associative learning paradigms that require multiple CS-US pairings, CTA

46 results in robust and rapid learning following one conditioning trial (Bures, Bermúdez-Rattoni, \&

47 Yamamoto, 1998; Reilly \& Schachtman, 2008). This feature of CTA learning makes it an ideal

48 paradigm to explore how differences in conditioning strength might alter the temporal dynamics of

49 memory generalization. The generalized aversion resulting from CTA conditioning is a well-

50 documented phenomenon (Domjan, 1975; Parker \& Revusky, 1982; Richardson, Williams, \& Riccio,

51 1984; Smith \& Theodore, 1984; Frank \& Nowlis, 1989; Chotro \& Alonso, 1999; Heyer et al., 2003;

52 Baird, St. John, \& Nguyen, 2005; Smith et al., 2012; Angulo, 2018; Wu, Ramos, et al., 2021), but it

53 is unclear how long this generalization can persist, which factors impact its duration, and the cellular

54 basis of this persistence.

55 Previously, we found that moderate conditioning (using a lower concentration of LiCl) induced a

56 generalized aversion that reversed within 24 hours, while strong conditioning (using a higher 
concentration of $\mathrm{LiCl}$ ) produced a generalized aversion that was still present at the 24 hours' time

point (Wu, Ramos, et al., 2021). Here, we explored this more persistent generalized aversion to determine how long it lasts, and the cellular basis of its persistence. We discovered that strong aversive conditioning results in a long-lasting generalized aversion that persisted for up to two-weeks and only reversed after experience with the generalized tastant. Next, we sought to characterize the cellular basis of its expression. We utilized the robust activity marking (RAM; Sørensen et al., 2016) system to label ensembles of neurons within gustatory cortex (GC) that were active during conditioning, and then performed ex-vivo whole-cell brain slice electrophysiology to measure excitatory postsynaptic strengths. Previous work has demonstrated the importance of the GC in CTA memory generalization (Kiefer \& Braun, 1977; Kiefer \& Braun, 1979; Wu, Ramos, et al., 2021). Our experiments revealed a potentiation of synaptic strengths occurring uniformly across the superficial and deep layers of GC in animals that had undergone strong CTA conditioning. Moreover, reversal of the long-lasting generalized aversion correlated with a reversal of the increases in synaptic strength. Unlike the CTA-induced potentiation that was observed uniformly across layers of GC, the exposure-induced reversal of synaptic potentiation occurred selectively in the superficial layers of GC. Together these findings illuminate the synaptic plasticity mechanisms that support a persistent long-lasting generalized aversion.

\section{Materials and Methods}

\subsection{Experimental subject details}

All experimental procedures were approved by Brandeis University Institutional Animal Care and Use Committee and followed the National Institute of Health guidelines for the Care and Use of Laboratory Animals. Young Long-Evans rats p28-p34 of both sexes were used in these experiments. Timed pregnant rats were obtained from Charles River Laboratories, and the progeny were maintained in the Foster Biomedical Research Labs at Brandeis University. After weaning at p21, littermates were individually housed in a humidity- and temperature-controlled environment and

82 entrained to a 12-hour light-dark cycle (light phase from 7:00-7:00) with ad libitum access to food 83 and water unless described otherwise. All subjects selected for electrophysiology experiments were 84 age matched to animals selected for behavioral experiments.

\subsection{Behavior}




\subsubsection{Two-bottle paradigm}

87 This CTA behavioral paradigm was conducted as previously described (Wu, Ramos, et al., 2021). Animals were transferred into individual home cages at p21 and habituated to two bottles with ad libitum access to water for three days. Next, the animals underwent water restriction for an additional three days, during which the access to water was limited to two hours. On the fourth day of restriction, rats were subjected to CTA conditioning. They received two bottles that contained the conditioned stimulus (CS, $10 \mathrm{mM}$ saccharin), for thirty minutes, followed by an intraperitoneal injection of the unconditioned stimulus (US, $\mathrm{LiCl}$ ). For moderate conditioning we used $0.15 \mathrm{M} \mathrm{LiCl}$ and for strong conditioning we used $0.30 \mathrm{M} \mathrm{LiCl}$, dosed at $1 \mathrm{ml} / 100 \mathrm{~g}$ body weight ( $1 \%$ body weight). After conditioning, the water restriction schedule was continued until testing. For the two-bottle test, rats were given one bottle of tastant and one bottle of water, for thirty minutes. The location of the two bottles was counterbalanced across presentations to prevent any positional biases. The results were quantified using a tastant preference score (TPS):

$$
T P S=\frac{\text { total tastant consumed }}{\text { total consumed }} \times 100
$$

100 To test for a generalized aversion (Gen. test), salt (150 $\mathrm{mM} \mathrm{NaCl})$ was used as a tastant. To measure

101 the reversal and attenuation of the generalized aversion, Gen. tests were conducted daily for three

102 days. After Gen. testing was complete, rats were given a CTA test to ensure that they had indeed

103 learned an aversion to the CS. All consumption was documented throughout the course of this

104 behavior to ensure that daily fluid intake was stable. No rats were excluded.

\section{$105 \quad 2.3 \quad$ RAM labeling of conditioning-active neurons}

\subsubsection{Viral vector}

107 RAM-dtTA-TRE-tdTomato was packaged in AAV serotype 9 by Duke Viral Vector Core.

\subsubsection{Virus surgery}

109 Rats were anesthetized via intraperitoneal infusion of a cocktail containing ketamine $(70 \mathrm{mg} / \mathrm{kg})$, 110 xylazine hydrochloride $(3.5 \mathrm{mg} / \mathrm{kg})$, and acepromazine maleate $(0.7 \mathrm{mg} / \mathrm{kg})$, and placed onto a 111 stereotaxic apparatus. The stereotaxic apparatus was localized over a heating pad to maintain the

112 animals body temperature. The skull was exposed and craniotomies were made above gustatory 113 cortex (GC) using the following coordinates: anterior-posterior with reference to bregma: $1.0 \mathrm{~mm}$; 
114 medial-lateral: $\pm 4.7 \mathrm{~mm}$; dorsal-ventral with reference to the brain surface: $3.6 \mathrm{~mm}$. RAM virus (400

$115 \mathrm{~nL}$ ) was unilaterally microinjected into GC through a glass micropipette connected to a

116 micromanipulator (Narishige, MO-10), at a rate of approximately $200 \mathrm{nl} / \mathrm{min}$. To allow adequate

117 diffusion of virus particles, the pipet remained in place for additional 3 minutes after injection and

118 was slowly withdrawn from the site. Injections were counterbalanced across hemispheres and both

119 hemispheres are equally represented in the data.

\section{$120 \quad$ 2.3.3 Labeling of conditioning-active neurons}

121 Customized chow containing low-dose doxycycline (40 ppm, ScottPharma) was added to the home

122 cage one day before virus surgery, and rats were maintained on doxycycline until CTA conditioning.

123 The doxycycline-containing chow was removed and replaced with regular chow one day before the

124 conditioning trial to allow adequate RAM induction. Two hours after the conditioning trial, rats were

125 placed back on the doxycycline diet to prevent further RAM activation. Acute brain slices were

126 collected 3 days after conditioning.

\section{$127 \quad 2.4 \quad$ Electrophysiology}

\section{$128 \quad$ 2.4.1 Ex-vivo acute brain-slice preparation}

129 Brain slices were produced following our previously documented protocols (Miska et al., 2018; Cary

130 \& Turrigiano, 2021; Wu, Ramos, et al., 2021). Briefly, rats (p28-p34) were anesthetized with

131 isoflurane, decapitated, and the brain was swiftly dissected out into ice cold carbogenated (95\% O2,

$1325 \% \mathrm{CO} 2$ ) standard ACSF (in mM: 126NaCl, $25 \mathrm{NaHCO} 3,3 \mathrm{KCl}, 2 \mathrm{CaCl} 2,2 \mathrm{MgSO} 4,1 \mathrm{NaH} 2 \mathrm{PO} 4$,

$1330.5 \mathrm{Na}$-Ascorbate, osmolarity adjusted to 310-315 mOsm with dextrose, $\mathrm{pH}$ 7.35). Coronal brain

134 slices $(300 \mathrm{~mm})$ containing GC were obtained from virus injected hemispheres using a vibratome

135 (LeicaVT1000). The slices were immediately transferred to a warm $\left(34^{\circ} \mathrm{C}\right)$ chamber filled with a

136 continuously carbogenated 'protective recovery' choline-based solution (in mM: $110 \mathrm{Choline-Cl,} 25$

$137 \mathrm{NaHCO}$, 11.6 Na-Ascorbate, $7 \mathrm{MgCl} 2,3.1 \mathrm{Na}-P y r u v a t e, 2.5 \mathrm{KCl}, 1.25 \mathrm{NaH} 2 \mathrm{PO}$, and $0.5 \mathrm{CaCl}$,

138 osmolarity 310-315 mOsm, pH 7.35) for 10 minutes (Ting et al., 2014). After incubation in choline,

139 the slices were transferred back to warm $\left(34^{\circ} \mathrm{C}\right)$ carbogenated standard ACSF and incubated another

14045 minutes. Brain slices were used for electrophysiology experiments between 1 - 7 hours post-

141 slicing. 


\subsubsection{Whole-cell patch clamp recording}

143 Slices were visualized on an Olympus upright epifluorescence microscope using a 10x air $(0.13$

144 numerical aperture) and 40x water-immersion objective (0.8 numerical aperture) with infrared-

145 differential interference contrast optics and an infrared CCD camera. Gustatory cortex was identified

146 in acute slices using the shape and morphology of the corpus callosum, piriform cortex and the lateral

147 ventricle as a reference. The borders of GC were determined by comparing the aforementioned

148 landmarks in slice to the Paxinos and Watson rat brain atlas. Pyramidal neurons from superficial and

149 deep layers across the dorsal-ventral axis of GC were visually targeted and identified by the presence

150 of an apical dendrite and teardrop shaped soma. In experiments involving the expression of a viral

151 construct, such as RAM, fluorophore expression was used to visually target pyramidal neurons. Virus

152 expression was consistent across layers (Wu, Ramos, et al., 2021). Pyramidal morphology was

153 confirmed by post hoc reconstruction of biocytin fills. Borosilicate glass recording pipettes were

154 pulled using a Sutter P-97 micropipette puller, with acceptable tip resistances ranging from 3 to 6

$155 \mathrm{M} \Omega$. All recordings were performed on submerged slices, continuously perfused with carbogenated

$15635^{\circ} \mathrm{C}$ recording solution. Data were low-pass filtered at $10 \mathrm{kHz}$ and acquired at $10 \mathrm{kHz}$ with

157 Axopatch 700B amplifiers and CV-7B headstages (Molecular Devices, Sunnyvale CA). Data were

158 acquired using WaveSurfer v0.953 (Janelia Research Campus), and all post hoc data analysis was

159 performed using in-house scripts written in MATLAB (Mathworks, Natick MA).

\section{$160 \quad$ 2.4.3 mEPSC recordings}

161 Cs + Methanesulfonate-based internal recording solution was used as previously reported (Miska et 162 al., 2018; Wu, Ramos, et al., 2021). This Cs+ internal contained (in mM) 115 Cs-Methanesulfonate, 16310 HEPES, 10 BAPTA•4Cs, 5.37 Biocytin, 2 QX314 Cl, 1.5MgCl2, 1 EGTA, 10 Na2-

164 Phosphocreatine, 4 ATP-Mg, and 0.3 GTP-Na, with sucrose added to bring osmolarity to $295 \mathrm{mOsm}$, 165 and $\mathrm{CsOH}$ added to bring $\mathrm{pH}$ to 7.35. For these recordings, pyramidal neurons were voltage clamped 166 to $-70 \mathrm{mV}$ in standard ACSF containing a drug cocktail of TTX (0.2mM), APV (50mM), PTX

167 (25mM). Traces of $10 \mathrm{~s}$ were acquired over a period of $\sim 10-15$ minutes allowing for the cell to fill for 168 later morphological verification. Neurons were excluded from analysis if Rs $>25 \mathrm{M} \Omega$ or if was Rin $>$ $1692 \sigma$ above the mean.

\section{$170 \quad 2.4 .4$ mEPSC analysis}

171 To reliably detect mEPSC events and limit selection bias, we used in-house software that employs a

172 semi-automated template-based detection method (Miska et al., 2018; Cary \& Turrigiano, 2021). 
173 Event inclusion criteria included amplitudes greater than $5 \mathrm{pA}$ and rise times less than $3 \mathrm{~ms}$. The

174 resulting events detected by our software were visually assessed posthoc and a subset of artefactual

175 events were excluded. The experimenter was blinded to experimental condition and treatment until

176 after the analysis was complete.

\section{$177 \quad 2.4 .5$ Recording from the conditioning-active ensemble}

178 Slices were collected exactly 3-days post-conditioning using the methods described above. mEPSCs 179 were recorded using the method described above. Fluorescent $\mathrm{RAM}^{+}\left(\right.$tdTomato $\left.^{+}\right)$cells were targeted 180 in both superficial and deep layers, where expression was equally robust (Wu, Ramos, et al., 2021).

181 The expression, or lack of, tdTomato was confirmed post hoc through immunostaining of the cells 182 using antibodies described in the immunohistochemistry section.

\subsection{Immunohistochemistry}

\subsubsection{Immunostaining of biocytin-filled cells}

185 After recording, slices were incubated in cold 4\% PFA for two days to fix the tissue. Following

186 fixation, slices were washed six times with PBS, preincubated with blocking buffer (5\% goat serum, $1873 \% \mathrm{BSA}, 0.3 \%$ Triton X-100 in PBS) for three hours at room temperature, and then incubated with 188 primary antibodies diluted in diluent buffer (5\% goat serum, $3 \% \mathrm{BSA}$ in PBS) at $4^{\circ} \mathrm{C}$ overnight. RAM 189 expression was verified using rabbit polyclonal anti-RFP (1:1000; Rockland 600-401-379). Slices 190 were counterstained with mouse monoclonal anti-NeuN (1:500; Millipore MAB-377). Following

191 incubation with primary antibodies, slices were washed six times with PBS and then incubated at $4^{\circ} \mathrm{C}$ 192 overnight with secondary antibodies diluted in diluent buffer. The secondary antibodies used for 193 these experiments were goat polyclonal anti-rabbit Alexa Fluor 568 (1:500; Thermo-Fisher, A194 11036) and goat polyclonal anti-mouse Alexa Fluor 647 (1:500; Thermo-Fisher, A-21236). Biocytin 195 fills were recovered by staining with streptavidin Alexa Fluor 488 (1:350; Thermo-Fisher, S-11223). 196 After incubation with secondary antibodies, slices were washed and mounted for imaging. Images 197 were acquired using the Leica SP5Laser Scanning Confocal Microscope.

\subsection{Quantification and statistics}

199 For all experiments including behavior and electrophysiology, individual experimental distributions 200 were tested for normality using the Anderson-Darling test. All experimental conditions passed the 201 normality test. A one sample, or two sample t test, or one-way ANOVA were used where 
appropriate. Significant ANOVA tests were followed by Tukey-Kramer post hoc comparisons.

Results of all statistical tests can be found in the figure legends. For behavior experiments $\mathrm{n}=$ number of animals, while for electrophysiology experiments $n=$ number of cells. Electrophysiology data were collected from at least 4 animals for each condition. Scatterplots were generated using publicly available MATLAB code (Ramirez, 2020).

\section{$207 \quad 3 \quad$ Results}

\subsection{Strong aversive conditioning triggers a long-lasting generalized aversion}

209 We previously demonstrated that strong CTA conditioning results in a generalized aversion that 210 could be observed at 24 hours post-conditioning (Wu, Ramos, et al., 2021), but it was unclear 211 whether this generalized aversion persists beyond this time point. Therefore, we first sought to 212 delineate how long the generalized aversion induced by strong CTA persists. We took animals

213 through strong CTA conditioning (strong CTA, 0.30M LiCl) or, as a control, a more moderate 214 conditioning regime (moderate $\mathrm{CTA}, 0.15 \mathrm{M} \mathrm{LiCl}$ ) which only induces a transient generalized 215 aversion that subsides within the first 24 hours post-conditioning (Wu, Ramos, et al., 2021). We then 216 tested for a generalized aversion 3 days post-conditioning (Figure 1A). We found that strong CTA

217 training resulted in a significant generalized aversion to salt at 3 days post-conditioning compared to

218 moderate CTA controls (Figure 1B). Repeated presentations of salt in the strong CTA group rapidly 219 reversed the generalized aversion, attenuating after just one presentation (Figure 1C). By contrast, in

220 the moderate CTA group which does not exhibit a long-lasting generalized aversion, repeated

221 presentation of salt caused no significant change in tastant preference score (Figure 1D). The 222 expression of the generalized aversion, as well as its reversal, did not impact the specific taste 223 aversion to the conditioned tastant, as animals still showed a strong aversion to saccharin after 224 generalization tests were completed (Figure 1E). These findings are consistent with our previous 225 results at the 24-hour time point for both experimental conditions (Wu, Ramos, et al., 2021).

226 Subsequent experiments revealed the long-lasting nature of the generalized aversion. We found that 227 we could observe a generalized aversion resulting from strong CTA conditioning at 7-, 10-, and 14228 days post-conditioning (Figure 2A \& 2B). In all instances, the generalized aversion reversed after 229 one trial with the generalized tastant without impacting the specific taste aversion to saccharine

230 (Figure 2B \& 2C). Together, these results demonstrate that increasing the strength of conditioning 231 with a higher concentration of $\mathrm{LiCl}$ triggers a long-lasting generalized aversion that persists up to two 232 weeks post-conditioning and only reverses after experience with the generalized tastant. 


\subsection{Strong aversive conditioning drives a long-lasting increase in postsynaptic strength}

234 We next endeavored to find an electrophysiological correlate for this long-lasting generalized 235 aversion within the gustatory cortex. Previously, we found that in the conditioning-active GC

236 neurons, moderate CTA conditioning produced a transient increase in postsynaptic strengths that was

237 homeostatically scaled down, while strong CTA conditioning resulted in a more sustained increase of

238 postsynaptic strengths (Wu, Ramos, et al., 2021). Notably, the presence or absence of this increase in

239 postsynaptic strengths correlated with the presence or absence of the generalized aversion. We thus

240 hypothesized that a long-lasting increase in postsynaptic strengths could support the long-lasting

241 generalized aversion induced by strong CTA conditioning. To test this, we virally expressed the

242 robust activity marking (RAM) system in GC to label the conditioning-activated neuronal ensemble

243 through the activity dependent expression of tdTomato (Sørensen et al., 2016; Wu, Ramos, et al.,

244 2021). RAM labeling is inhibited by doxycycline (Dox); by removing Dox we could restrict labeling

245 to a small window during which animals underwent either strong or moderate CTA conditioning.

246 Congruent with the behavioral measurements in Figure 1, brain slices were prepared 3 days after

247 CTA induction for electrophysiological recording (Figure 3A). We then targeted either RAM

248 (tdTomato $^{+}$) or nearby $\mathrm{RAM}^{-}\left(\right.$tdTomato $\left.^{-}\right)$pyramidal neurons for whole-cell electrophysiological

249 recordings (Figure 3B) and measured miniature excitatory postsynaptic currents (mEPSCs) to

250 quantify postsynaptic strength (Figure 3C). We found that strong CTA conditioning produced a

251 significant increase in mEPSC amplitude in RAM $^{+}$neurons; more surprisingly, a similar increase was

252 observed in RAM $^{-}$neurons when compared to neurons recorded from moderate CTA animals

253 (Figure 3D \& 3E). Other studies have similarly documented changes in synaptic strength in GC

254 neurons using whole-cell physiology following CTA learning without an activity-dependent labeling

255 scheme, suggesting that synaptic plasticity occurs across a large population of cells in GC (Haley et

256 al., 2020). These findings reveal that strong CTA conditioning produces a long-lasting increase in

257 postsynaptic strengths that parallels the long-lasting generalized aversion and suggest a possible GC-

258 wide induction of synaptic plasticity. Because neurons can be RAM- either because they did not

259 express the virus, or because they did but were not active during conditioning, for the remainder of

260 our experiments we focused solely on $\mathrm{RAM}^{+}$neurons to ensure we recorded from a more

261 homogenous population of cells.

$262 \quad 3.3$ Experience with salt rapidly reverses increases in postsynaptic strength 
263 If the increase in postsynaptic strength contributes to the expression of the long-lasting generalized

264 aversion, then reversal of the generalized aversion by salt exposure should also reverse the increases

265 in mEPSC amplitude in conditioning-active GC neurons. To test this hypothesis, animals were

266 exposed to salt and then 2- or 24-hours following salt exposure, slices were made for

267 electrophysiological recordings (Figure 4A). As in the previous experiments, at the time of

268 recording, 3 days had elapsed post-conditioning. These experiments revealed that, when compared to

269 the average baseline amplitude following strong CTA conditioning in Figure 3 (green dashed line),

270 experience with the generalized tastant (salt) rapidly decreased mEPSC amplitude. This depression

271 was evident as early as 2 hours following salt presentation and was greater in magnitude after 24

272 hours (Figure 4B). In contrast, when compared to the average baseline amplitude following moderate

273 CTA conditioning in Figure 3 (green dashed line), animals in the moderate CTA group showed no

274 changes at 2- or 24-hours post-salt exposure (Figure 4C). These findings reveal a strong correlation

275 between postsynaptic strength and memory generalization within the conditioning-active ensemble.

276 Furthermore, they suggest an important contribution from forms of synaptic depression that operate

277 over a time scale of hours.

$278 \quad 3.4$ CTA-induced increase in postsynaptic strength occurs uniformly across superficial and 279 deep layers of GC

280 It is known that superficial (layers II/III) and deep (layers V/VI) layers of the gustatory cortex are 281 innervated differentially by structures important for CTA learning (Allen et al., 1991; Nakashima et 282 al., 2000; Maffei, Haley, \& Fontanini, 2012; Haley, Fontanini, \& Maffei, 2016). This raised the 283 question of whether synaptic strengthening following CTA learning is uniformly expressed across the 284 different layers of gustatory cortex. The recordings presented in Figure 3 were performed across 285 superficial and deep layers of the GC (Figure 5A). When we sorted these data based on layers, we 286 found that strong CTA conditioning indeed increased mEPSC amplitude (compared to moderate

287 CTA controls) in both superficial and deep layers (Figure 5B). Thus, while these different layers are 288 distinctly innervated, strong CTA induces widespread and uniform changes in synaptic plasticity 289 across the cortical layers of GC.

\subsection{Reversal is more pronounced in superficial layers of GC}

291 We next asked if the reversal of postsynaptic strengths after salt exposure also occurred uniformly 292 across superficial and deep layers. We sorted the dataset in Figure 4 by layers and normalized each 
group after salt exposure to the corresponding average amplitude without salt exposure (green dashed line). This revealed that neurons in the superficial layers of gustatory cortex exhibited a reversal of mEPSC amplitude after salt exposure (Figure 5C). In contrast, although strong CTA induced an increase in mEPSC amplitude across both superficial and deep layers, the reduction in mEPSC amplitude after salt exposure was less pronounced in the deep layers and did not reach statistical significance (Figure 5D). In animals that underwent moderate CTA conditioning, there were little-tono changes in mEPSC amplitude. In the superficial layers, there was a reduction at 2 hours that was gone at 24 hours, and in the deep layers there was no change at either time point (Figure 5E \& 5F). Together these results indicate that reversal of postsynaptic strengths induced by salt exposure is a layer-specific process that primarily occurs within the superficial layers of GC.

\section{Discussion}

304 Understanding the cellular basis of memory generalization can yield important insights into the maladaptive, persistent overgeneralization that is characteristic of anxiety disorders. Here we took advantage of CTA learning's one trial nature to study a long-lasting generalized aversion resulting from a single strong conditioning event. In doing so, we uncovered changes in postsynaptic strength occurring in a layer specific manner within the gustatory cortex that correlated with many of our behavioral observations. Our experiments revealed that postsynaptic strength in the superficial layers of GC seems to increase, then decrease, with the presence and then reversal of the long-lasting generalized aversion, respectively. Interestingly, postsynaptic strength in the deep layers of GC also increases after CTA learning, but does not significantly change as the generalized aversion reverses,

313 but instead persists much like the specific taste aversion to saccharin. These data suggest that rather

314 than being functionally homogenous, neurons within the conditioning active ensemble in GC

315 subserve different memory functions depending on what layer they are found in. These results are in

316 line with the functionally distinct engram ensembles found in the hippocampus that govern the

317 balance between memory generalization and specificity (Sun et al., 2020). Future experiments are

318 needed to identify exactly what form of synaptic plasticity is responsible for the CTA-induced

319 strengthening, the synaptic depression induced by exposure to the generalized tastant, and whether

320 these forms of synaptic plasticity are causally involved in this behavior.

321 In these experiments we leveraged of our ability to manipulate the strength of the learned aversion by

322 changing the concentration of $\mathrm{LiCl}$ and taking animals through a strong aversive conditioning

323 scheme $(0.30 \mathrm{M} \mathrm{LiCl}, 1 \%$ b.w. $)$. This concentration and dosage of $\mathrm{LiCl}$ has been previously 
324 demonstrated to result in a maximum aversion to the CS (Nachman \& Ashe, 1973). Using this

325 approach, we found that after a single conditioning trial, animals can form a long-lasting generalized

326 aversion that persists up to two weeks post-CTA training. This persistence, to our knowledge, is

327 longer than any reported thus far in the CTA literature on generalization (Domjan, 1975; Parker \&

328 Revusky, 1982; Richardson, Williams, \& Riccio, 1984; Smith \& Theodore, 1984; Frank \& Nowlis,

329 1989; Chotro \& Alonso, 1999; Heyer et al., 2003; Baird, St. John, \& Nguyen, 2005; Smith et al.,

330 2012; Angulo, 2018). The ethological significance of this behavior and the mechanism by which this

331 generalization is occurring remain to be worked out. One possibility is that strong aversive

332 conditioning results in a state of long-lasting enhanced caution towards novel stimuli to protect the

333 animal from further toxicosis. This may reflect toxicosis induced enhanced neophobia, where CTA

334 produces enhancement to an already decreased intake of unfamiliar, novel stimuli (Domjan, 1975;

335 Reilly \& Schachtman, 2008; Lin et al., 2012). In the context of associative learning, generalization is

336 thought to occur more easily across stimuli that share "elements" with each other (McLaren \&

337 Mackintosh, 2002). But what these elementary features are with respect to tastants and CTA is

338 unknown. In addition to basic taste modality (sweet, sour, salty, etc....) the GC has been shown to

339 encode other taste features such as identity, palatability, and novelty/familiarity, all of which are

340 elements to potentially evaluate taste stimuli by (Gallo, Roldan, \& Bures, 1992; Lin et al., 2012;

341 Flores et al., 2018; Mukherjee, Wachutka, \& Katz, 2019). In our study, what elementary features are

342 shared by salt, the generalized tastant, and saccharin, the conditioned stimulus, remain to be

343 identified. The results of future experiments will further our understanding regarding whether this is

344 truly stimulus generalization between saccharin and salt, or an avoidance like behavior similar to

345 enhanced neophobia.

346 Our electrophysiology experiments found that the long-lasting generalized aversion resulting from

347 strong CTA correlated with a similarly persistent increase in postsynaptic strengths in both RAM $^{+}$

348 and $\mathrm{RAM}^{-}$cells. There are several possible interpretations regarding why $\mathrm{RAM}^{-}$cells, many of which

349 are presumably not activated during conditioning, also exhibit an increase in postsynaptic strengths

350 post-conditioning. One possibility is that these cells could be infected with RAM virus, but

351 expression of tdTomato is below the threshold required for detection during live-cell recording. This

352 is unlikely because in all our experiments, cells were recovered post-hoc using biocytin fills and

353 stained against tdTomato, which would amplify the signal and allow us to detect even very low levels

354 of RAM expression. Another possibility is that these cells are not transfected with the virally

355 expressed RAM system and are activated by conditioning. We cannot exclude this possibility using 
our current methodology. However, it is unlikely that all $\mathrm{RAM}^{-}$neurons fall into this category, given that our recordings were performed on neighboring $\mathrm{RAM}^{+}$and $\mathrm{RAM}^{-}$neurons within cortical areas showing high tdTomato expression. The last possibility is that these are cells infected with RAM virus, but not activated during conditioning (true $\mathrm{RAM}^{-}$). It is possible that $\mathrm{RAM}^{-}$cells are later recruited during memory consolidation, thereby manifesting changes in synaptic strength. CTA conditioning is known to induce dynamic changes in firing rate within GC, which may suggest that different neuronal populations may undergo changes in synaptic plasticity on distinct time scales post-conditioning and this temporal activation facilitates the formation of an aversive memory (Moran \& Katz, 2014; Arieli, Younis, \& Moran, 2021). Additionally, previous studies have documented changes in synaptic strength in GC neurons following CTA training without an activity dependent labeling scheme, suggesting that synaptic plasticity occurs across a large population of cells in GC (Haley et al., 2020). Taken together, these findings hint at the possibility of GC-wide induction of synaptic plasticity following CTA conditioning. The strong correlation between generalized aversion and this strengthening, including the ability to reverse both by salt exposure, suggests that this strengthening could be causally involved in the expression of the generalized aversion, but more experiments are needed to establish causality.

Our previous experiments revealed that synaptic downscaling of postsynaptic strengths was important for reversing the transient generalized aversion resulting from moderate CTA training, and establishing CTA specificity (Wu, Ramos, et al., 2021). Whether a similar mechanism is involved in the experience dependent reversal of the long-lasting generalized aversion is unknown. The experiments presented in Figure 4 demonstrate that as soon as 2 hours following salt exposure, mEPSC amplitudes are significantly decreased. The decrease in mEPSC amplitude is more pronounced 24 hours after salt exposure, suggesting that this depotentiation of postsynaptic strengths is initiated rapidly and continues to unfold slowly over the course of 24 hours. No changes were observed following salt exposure in animals that underwent moderate CTA conditioning. These data reveal a strong correlation between the experience dependent reversal of the long-lasting generalized aversion and reversal of the increases in postsynaptic strength following salt exposure. Several forms of synaptic plasticity could potentially drive this decrease in postsynaptic strength. First, previous research has demonstrated that LTD is involved in the extinction of CTA (Li et al., 2016), and salt exposure may induce LTD in a similar fashion during reversal of the generalized aversion. 
this scenario, downscaling becomes "gated" by future tastant experiences. Recent research on the behavioral-state gating of homeostatic plasticity supports this possibility (Hengen et al., 2016; Torrado Pacheco et al., 2021). Future experiments that use antagonists targeting specific forms of synaptic plasticity will clarify the mechanisms that drive the reversal of postsynaptic strengths after

392 salt exposure and could reveal important insights into how we can reverse or weaken more persistent

393 forms of memory generalization.

394 GC consists of superficial and deep layers that receive distinct projections from sub-cortical nuclei

395 important for CTA learning. For example, inputs from the gustatory thalamus have been shown to be 396 uniformly diffused across the GC, with a slight bias for the granular layer (layer IV) and layer V

397 (Allen et al., 1991; Nakashima et al., 2000; Maffei, Haley, \& Fontanini, 2012). In contrast, the 398 amygdala projects to both superficial and deep layers of GC, but the magnitude of these inputs is 399 larger in the superficial layers (Haley, Fontanini, \& Maffei, 2016). Compared to other sensory 400 cortices, such as visual cortex, little work has been done to characterize the synaptic properties of 401 distinct inputs into distinct layers of GC (Maffei, Haley, \& Fontanini, 2012). Even less is known 402 about how these different inputs are modulated by CTA learning and whether there exist layer specific motifs. Our finding that strong CTA conditioning produces increases in postsynaptic strengths in $\mathrm{RAM}^{+}$and $\mathrm{RAM}^{-}$neurons in both superficial and deep layers further supports the notion that CTA results in a GC-wide induction of synaptic plasticity. During reversal, the reduction in postsynaptic strength is more pronounced in superficial layers of GC. In comparison, little to no change was observed across layers in the moderate CTA animals following salt exposure. Altogether, these data suggest that while CTA learning induces region-wide changes in postsynaptic strengths, a layer specific plasticity mechanism may be responsible for the establishment and reversal of the generalized aversion. Given that superficial layers receive inputs from multiple subcortical nuclei, it will require further experiments to isolate specific populations of synaptic inputs in which the generalized tastant-induced changes in synaptic plasticity occur.

413 Experiments on short- and long-term memory have previously revealed that these different types of 414 memory, operating over distinct time scales, produce short- and long-term changes in synaptic 415 efficacy, respectively (Kandel et al., 2013; Kandel, Dudai, \& Mayford, 2014). Whether similar 416 mechanisms are at work in the context of memory generalization remained unknown. Our previous 417 experiments using moderate CTA conditioning revealed that a transient generalized aversion was 418 correlated with transient changes in synaptic strength, analogous to the short-term changes observed 
419 during short-term memory (Wu, Ramos, et al., 2021). Here, we found that the long-lasting

420 generalized aversion resulting from strong CTA correlated with a similarly long-lasting increase in

421 postsynaptic strengths, analogous to changes seen in long-term memory formation (Kandel, 2012;

422 Kandel, Dudai, \& Mayford, 2014; Takeuchi, Duszkiewicz, \& Morris, 2014). Finally, exposure to salt

423 rapidly reversed the generalized aversion without impacting the retrieval of CTA memory at later

424 time points, suggesting that the encoding of CTA and the generalized aversion either use distinct

425 mechanisms or occur in distinct populations of cells. Our data provides evidence for the latter and

426 suggests that the different layers of GC play functionally distinct roles in CTA memory. These

427 findings suggest an interesting hypothesis: that changes within the conditioning-active ensemble are

428 important for the initial acquisition of CTA memory, but after acquisition, the CTA conditioning-

429 active neuronal ensemble functionally diverges, and cells in the superficial layers instead work to

430 support the generalized aversion while cells in the deep layers are dedicated to the specific taste

431 aversion learned against the CS. This hypothesis is supported by evidence from studies in the

432 hippocampus where it was found that within an engram ensemble there exists functionally

433 heterogeneous populations of neurons that work to govern the balance of memory generalization and

434 specificity (Sun et al., 2020). Furthermore, these functionally distinct ensembles are genetically

435 defined based on their immediate early gene expression profile, either Npas 4 or Fos, both of which

436 are transcriptional pathways labeled by our RAM system (Sørensen et al., 2016). If this hypothesis is

437 correct, it will yield a new perspective regarding the CTA-conditioning active ensemble, CTA-

438 memory retrieval, and memory generalization.

\section{Data Availability Statement}

440 All data generated from these experiments are included in the figures and supplemental figures. Raw

441 data supporting the conclusions of this article will be made available by the authors upon request.

442 Custom MATLAB scripts used for the analyses of electrophysiology data can be found at

443 https://github.com/BrianAndCary/papers/tree/master/bcary2020_paper/mini_FI_GUI.

\section{Author Contributions}

445 These experiments were conceptualized by RR, CHW, and GGT. The behavior experiments were

446 carried out by RR and CHW. RR performed the electrophysiology experiments, immunofluorescence

447 staining, and analysis. RR wrote the original draft of this manuscript. RR, CHW, and GGT reviewed

448 and edited the manuscript together. 


\section{$449 \quad 7 \quad$ Funding}

450 This work was funded by NIH grant R35NS 111562 (GGT) and NS108506 (RR).

4518 Conflict of Interest

452 The authors declare that the research was conducted in the absence of any commercial or financial 453 relationships that could be construed as a potential conflict of interest.

\section{Acknowledgments}

455 We thank Don Katz, Bradly Stone, Jian-You Lin and members of the Turrigiano Lab for critical

456 feedback on these experiments. Additionally, we thank Arianna Maffei for constructive feedback on 457 an earlier draft of this manuscript. 


\section{References}

459 Allen, G. V., Saper, C. B., Hurley, K. M., \& Cechetto, D. F. (1991). Organization of visceral and

460 limbic connections in the insular cortex of the rat. The Journal of Comparative Neurology, 311(1), 1-

46116.

462

Angulo, R. (2018). Pre-exposure Schedule Effects on Generalization of Taste Aversion and

Palatability for Thirsty and Not Thirsty Rats. Frontiers in Psychology, 9, 878.

465

Arieli, E., Younis, N., \& Moran, A. (2021). Distinct progressions of neuronal activity changes underlie the formation and consolidation of a gustatory associative memory. The Journal of Neuroscience: The Official Journal of the Society for Neuroscience.

https://doi.org/10.1523/JNEUROSCI.1599-21.2021

https://doi.org/10.1007/bf01871001

Baird, J.-P., St John, S. J., \& Nguyen, E. A.-N. (2005). Temporal and qualitative dynamics of conditioned taste aversion processing: combined generalization testing and licking microstructure analysis. Behavioral Neuroscience, 119(4), 983-1003.

Bures, J., Bermúdez-Rattoni, F., \& Yamamoto, T. (1998). Conditioned taste aversion: Memory of a special kind. Oxford Psychology Series No. 31., 178.

Cary, B. A., \& Turrigiano, G. G. (2021). Stability of neocortical synapses across sleep and wake states during the critical period in rats. eLife, 10. https://doi.org/10.7554/eLife.66304

Chotro, M. G., \& Alonso, G. (1999). Effects of stimulus preexposure on the generalization of conditioned taste aversions in infant rats. Developmental Psychobiology, 35(4), 304-317. 
491 Domjan, M. (1975). Poison-induced neophobia in rats: Role of stimulus generalization of

492 conditioned taste aversions. Animal Learning \& Behavior, 3(3), 205-211.

493

494 Dunsmoor, J. E., \& Paz, R. (2015). Fear Generalization and Anxiety: Behavioral and Neural

495 Mechanisms. Biological Psychiatry, 78(5), 336-343.

Flores, V. L., Parmet, T., Mukherjee, N., Nelson, S., Katz, D. B., \& Levitan, D. (2018). The role of the gustatory cortex in incidental experience-evoked enhancement of later taste learning. Learning \& Memory, 25(11), 587-600.

Frank, M. E., \& Nowlis, G. H. (1989). Learned aversions and taste qualities in hamsters. Chemical Senses, 14(3), 379-394.

Gallo, M., Roldan, G., \& Bures, J. (1992). Differential involvement of gustatory insular cortex and amygdala in the acquisition and retrieval of conditioned taste aversion in rats. Behavioural Brain Research, 52(1), 91-97.

Haley, M., Bruno, S., Fontanini, A., \& Maffei, A. (2020). LTD at amygdalocortical synapses as a

Haley, M. S., Fontanini, A., \& Maffei, A. (2016). Laminar- and Target-Specific Amygdalar Inputs in

512 Rat Primary Gustatory Cortex. The Journal of Neuroscience: The Official Journal of the Society for

513 Neuroscience, 36(9), 2623-2637.

515 Heck, G. L., Mierson, S., \& DeSimone, J. A. (1984). Salt taste transduction occurs through an 516 amiloride-sensitive sodium transport pathway. Science, 223(4634), 403-405.

518 Hengen, K. B., Torrado Pacheco, A., McGregor, J. N., Van Hooser, S. D., \& Turrigiano, G. G. 519 (2016). Neuronal Firing Rate Homeostasis Is Inhibited by Sleep and Promoted by Wake. Cell, $520165(1), 180-191$. 
522 Heyer, B. R., Taylor-Burds, C. C., Tran, L. H., \& Delay, E. R. (2003). Monosodium glutamate and

523 sweet taste: generalization of conditioned taste aversion between glutamate and sweet stimuli in rats.

524 Chemical Senses, 28(7), 631-641.

525

526 Josselyn, S. A., \& Tonegawa, S. (2020). Memory engrams: Recalling the past and imagining the

527 future. Science, 367(6473).

Kandel, E. R. (2012). The molecular biology of memory: cAMP, PKA, CRE, CREB-1, CREB-2, and CPEB. Molecular Brain, 5, 14.

Kandel, E. R., Dudai, Y., \& Mayford, M. R. (2014). The molecular and systems biology of memory. Cell, 157(1), 163-186.

Kandel, E. R., Schwartz, J. H., Jessell, T. M., Siegelbaum, S. A., \& Hudspeth, A. J. (2013).

Principles of Neural Science, Fifth Edition. McGraw Hill Professional.

Kheirbek, M. A., Klemenhagen, K. C., Sahay, A., \& Hen, R. (2012). Neurogenesis and generalization: a new approach to stratify and treat anxiety disorders. Nature Neuroscience, 15(12), $1613-1620$.

542 Kiefer, S. W., \& Braun, J. J. (1977). Absence of differential associative responses to novel and

543 familiar taste stimuli in rats lacking gustatory neocortex. Journal of Comparative and Physiological

544 Psychology, 91(3), 498-507.

Kiefer, S. W., \& Braun, J. J. (1979). Acquisition of taste avoidance habits in rats lacking gustatory neocortex. Physiological Psychology, 7(3), 245-250.

Li, W.-G., Liu, M.-G., Deng, S., Liu, Y.-M., Shang, L., Ding, J., Hsu, T.-T., Jiang, Q., Li, Y., Li, F., Zhu, M. X., \& Xu, T.-L. (2016). ASIC1a regulates insular long-term depression and is required for the extinction of conditioned taste aversion. Nature Communications, 7, 13770.

553 Lin, J.-Y., Roman, C., Arthurs, J., \& Reilly, S. (2012). Taste neophobia and c-Fos expression in the 554 rat brain. Brain Research, 1448, 82-88. 
556 Maffei, A., Haley, M., \& Fontanini, A. (2012). Neural processing of gustatory information in insular 557 circuits. Current Opinion in Neurobiology, 22(4), 709-716.

Mahan, A. L., \& Ressler, K. J. (2012). Fear conditioning, synaptic plasticity and the amygdala: implications for posttraumatic stress disorder. Trends in Neurosciences, 35(1), 24-35.

McLaren, I. P. L., \& Mackintosh, N. J. (2002). Associative learning and elemental representation: II. Generalization and discrimination. Animal Learning \& Behavior, 30(3), 177-200. experience inversely regulates feedforward and feedback excitation-inhibition ratio in rodent visual cortex. eLife, 7. https://doi.org/10.7554/eLife.38846

Moran, A., \& Katz, D. B. (2014). Sensory cortical population dynamics uniquely track behavior across learning and extinction. The Journal of Neuroscience: The Official Journal of the Society for Neuroscience, 34(4), 1248-1257.

Nachman, M., \& Ashe, J. H. (1973). Learned taste aversions in rats as a function of dosage,

Nakashima, M., Uemura, M., Yasui, K., Ozaki, H. S., Tabata, S., \& Taen, A. (2000). An anterograde and retrograde tract-tracing study on the projections from the thalamic gustatory area in the rat: distribution of neurons projecting to the insular cortex and amygdaloid complex. In Neuroscience Research (Vol. 36, Issue 4, pp. 297-309). https://doi.org/10.1016/s0168-0102(99)00129-7 
Ramirez, M.L. (2020). UnivarScatter.https://github.com/manulera/UnivarScatter.

Reilly, S., \& Schachtman, T. R. (2008). Conditioned Taste Aversion: Neural and Behavioral

Processes. Oxford University Press.

Richardson, R., Williams, C., \& Riccio, D. C. (1984). Stimulus generalization of conditioned taste

Shepard, R. N. (1987). Toward a universal law of generalization for psychological science. Science, 237(4820), 1317-1323.

598

Smith, D. V., \& Theodore, R. M. (1984). Conditioned taste aversions: generalization to taste mixtures. Physiology \& Behavior, 32(6), 983-989.

601

Smith, K. R., Treesukosol, Y., Paedae, A. B., Contreras, R. J., \& Spector, A. C. (2012). Contribution of the TRPV1 channel to salt taste quality in mice as assessed by conditioned taste aversion generalization and chorda tympani nerve responses. American Journal of Physiology. Regulatory, Integrative and Comparative Physiology, 303(11), R1195-R1205.

Sørensen, A. T., Cooper, Y. A., Baratta, M. V., Weng, F.-J., Zhang, Y., Ramamoorthi, K., Fropf, R., LaVerriere, E., Xue, J., Young, A., Schneider, C., Gøtzsche, C. R., Hemberg, M., Yin, J. C., Maier, S. F., \& Lin, Y. (2016). A robust activity marking system for exploring active neuronal ensembles. eLife, 5. https://doi.org/10.7554/eLife.13918

611

612 Sun, X., Bernstein, M. J., Meng, M., Rao, S., Sørensen, A. T., Yao, L., Zhang, X., Anikeeva, P. O., \& 613 Lin, Y. (2020). Functionally Distinct Neuronal Ensembles within the Memory Engram. Cell, 181(2), 410-423.e17.

Takeuchi, T., Duszkiewicz, A. J., \& Morris, R. G. M. (2014). The synaptic plasticity and memory hypothesis: encoding, storage and persistence. In Philosophical Transactions of the Royal Society B: 
620 Ting, J. T., Daigle, T. L., Chen, Q., \& Feng, G. (2014). Acute Brain Slice Methods for Adult and

621 Aging Animals: Application of Targeted Patch Clamp Analysis and Optogenetics. In M. Martina \&

622 S. Taverna (Eds.), Patch-Clamp Methods and Protocols (pp. 221-242). Springer New York.

623

624 Torrado Pacheco, A., Bottorff, J., Gao, Y., \& Turrigiano, G. G. (2021). Sleep Promotes Downward 625 Firing Rate Homeostasis. Neuron, 109(3), 530-544.e6.

626

627 Wu, C.-H., Ramos, R., Katz, D. B., \& Turrigiano, G. G. (2021). Homeostatic synaptic scaling

628 establishes the specificity of an associative memory. Current Biology: CB, 31(11), 2274-2285.e5. 


\section{Figure Captions}

630 Figure 1. Strong aversive conditioning triggers a long-lasting generalized aversion. (A) Two-bottle

631 CTA learning paradigm for testing the persistence of the generalized aversion. (B) Gen. test; preference 632 score for salt tested 3 days post-conditioning (two-sample $t$ test, $p=0.0220$ ). (C) SCTA repeated Gen.

633 test; preference for salt tested at 3-, 4-, and 5-days post-conditioning (One-way ANOVA, p =0.0036;

634 Tukey-Kramer post-hoc tests, day 3 vs day 4, $\mathrm{p}=0.0184$; day 3 vs day 5, p =0.0047; day 4 vs day 5, p $635=0.8430$ ). (D) MCTA repeated Gen. test; preference for salt tested at 3-, 4-, and 5-days post636 conditioning (One-way ANOVA, p = 0.0716). (E) CTA test; preference score for saccharin of 3-day 637 experimental conditions (two-sample $\mathrm{t}$ test, $\mathrm{p}=0.4387$ ).

638 Figure 2. Strong CTA-induced generalized aversion can be observed up to two-weeks post639 conditioning (A) Two-bottle CTA learning paradigm for testing the persistence of the generalized 640 aversion at 7-, 10-, and 14-days post-conditioning. (B) Repeated Gen. test; preference score for $\mathrm{NaCl}$ 641 tested 7-, 10-, and 14-days experimental conditions. Each test is separated by a 24-hour interval. (C) 642 CTA test; preference score for saccharin of long-duration experimental conditions.

643 Figure 3. Strong aversive conditioning drives a long-lasting increase in postsynaptic strength. (A) 644 Experimental design. (B) Biocytin fills (cyan) of $\mathrm{RAM}^{+}$(magenta; white arrows) and RAM- (yellow arrows) pyramidal cells in gustatory cortex. (C) Representative mEPSC recordings. (D) Cell-average mEPSC amplitudes of RAM ${ }^{+}$cells 3 days post-conditioning (two-sample t test, $\mathrm{p}=0.0035$ ). (E) Cellaverage mEPSC amplitudes of $\mathrm{RAM}^{-}$cells 3 days post-conditioning (two-sample t test, $\mathrm{p}=0.0224$ ).

648 Figure 4. Experience with salt rapidly reverses increases in postsynaptic strength. (A) Experimental 649 design. (B) SCTA cell-average mEPSC amplitudes of $\mathrm{RAM}^{+}$cells 2- and 24-hours post-salt exposure. 650 Green dashed line represents average mEPSC amplitude from $\mathrm{RAM}^{+}$cells in SCTA no-salt exposure 651 conditions (Figure 3D; one-sample t test against hypothesized mean, 2 hours, p = 0.0018, 24 hours, $p$ $652=5.5750 \mathrm{e}-06)$. (C) MCTA cell-average mEPSC amplitudes of RAM ${ }^{+}$cells 2- and 24-hours post-salt 653 exposure. Green dashed line represents average mEPSC amplitude from $\mathrm{RAM}^{+}$cells in MCTA no-salt 654 exposure conditions (Figure 3D; one-sample t test against hypothesized mean, 2 hours, $p=0.0857,24$ 655 hours, $\mathrm{p}=0.7945)$.

656 Figure 5. Distinct layer processes govern the induction and reversal of generalization. (A) Biocytin 657 fills (cyan) of cells recorded from the superficial and deep layers of gustatory cortex. (B) Cell-average 658 mEPSC amplitudes of pooled $\mathrm{RAM}^{+}$and $\mathrm{RAM}^{-}$cells 3 days post-conditioning sorted by superficial 
659 and deep layers (superficial layers, two-sample t test, $\mathrm{p}=0.0104$; deep layers, two-sample $\mathrm{t}$ test, $\mathrm{p}=$ 660 0.0498). (C) SCTA cell-average mEPSC amplitudes of RAM ${ }^{+}$cells from superficial layers 2- and 24661 hours post-salt exposure. Green dashed line represents average mEPSC amplitude from RAM ${ }^{+}$cells 662 from superficial layers in SCTA no-salt exposure conditions (Figure 5B; one-sample t test against 663 hypothesized mean, 2 hours, $\mathrm{p}=0.0355,24$ hours, $\mathrm{p}=2.7205 \mathrm{e}-05$ ). (D) SCTA cell-average mEPSC 664 amplitudes of $\mathrm{RAM}^{+}$cells from deep layers 2- and 24-hours post-salt exposure. Green dashed line 665 represents average mEPSC amplitude from $\mathrm{RAM}^{+}$cells from deep layers in SCTA no-salt exposure 666 conditions (Figure 5B; one-sample t test against hypothesized mean, 2 hours, p = 0.1929, 24 hours, $p$ $667=0.2087)$. (E) MCTA cell-average mEPSC amplitudes of RAM $^{+}$cells from superficial layers 2- and 668 24-hours post-salt exposure. Green dashed line represents average mEPSC amplitude from RAM ${ }^{+}$cells 669 from superficial layers in MCTA no-salt exposure conditions (Figure 5B; one-sample t test against 670 hypothesized mean, 2 hours, $\mathrm{p}=0.0166,24$ hours, $\mathrm{p}=0.7606)$. (F) MCTA cell-average mEPSC 671 amplitudes of $\mathrm{RAM}^{+}$cells from deep layers 2- and 24-hours post-salt exposure. Green dashed line 672 represents average mEPSC amplitude from $\mathrm{RAM}^{+}$cells from deep layers in MCTA no-salt exposure 673 conditions (Figure 5B; one-sample $t$ test against hypothesized mean, 2 hours, $p=0.7262,24$ hours, $p$ $674=0.5056)$. 
A

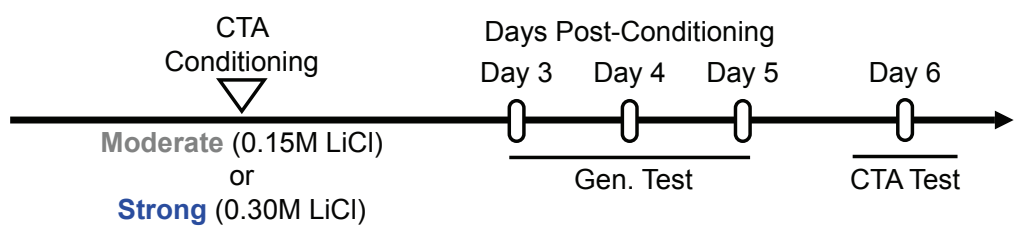

C

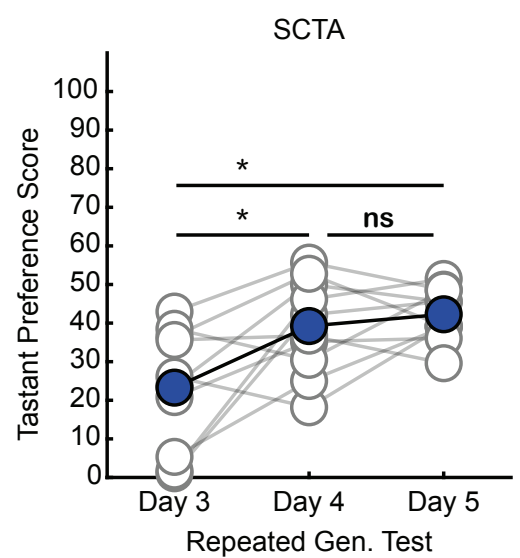

B

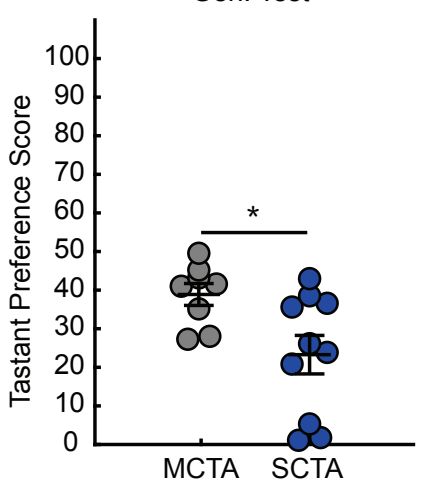

D

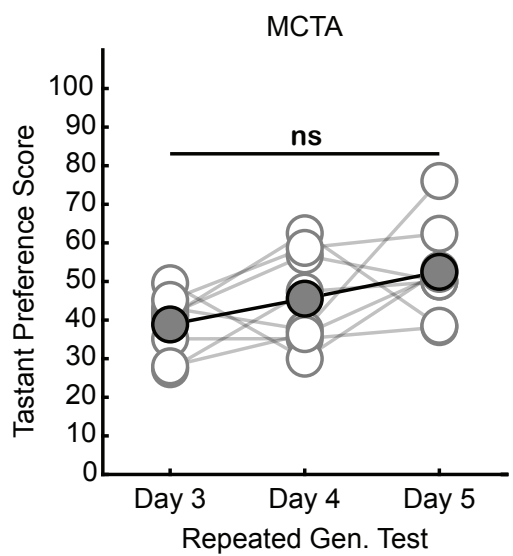

E

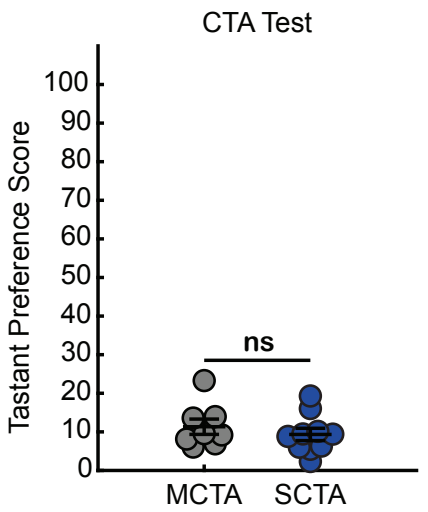




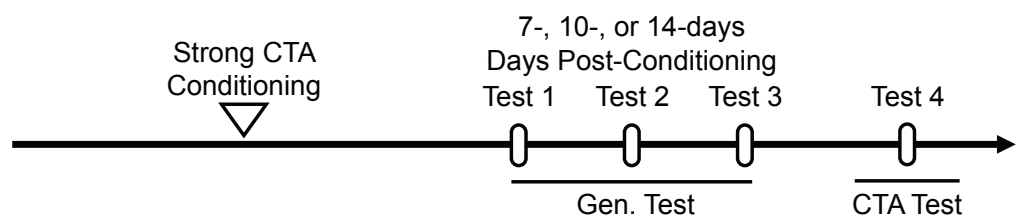

B

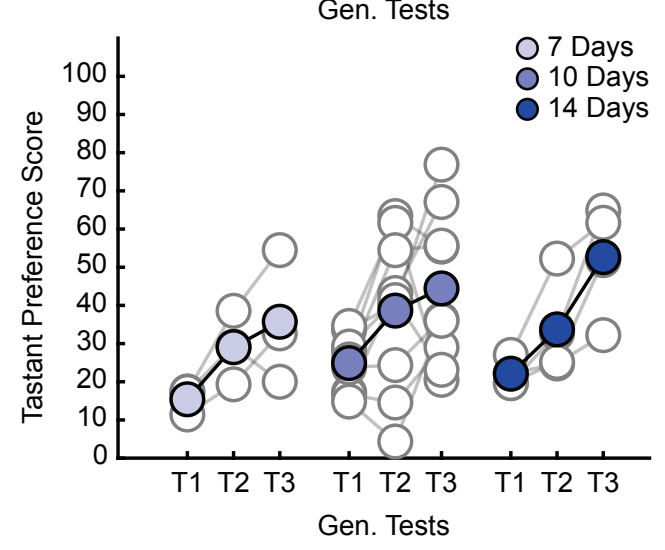

C

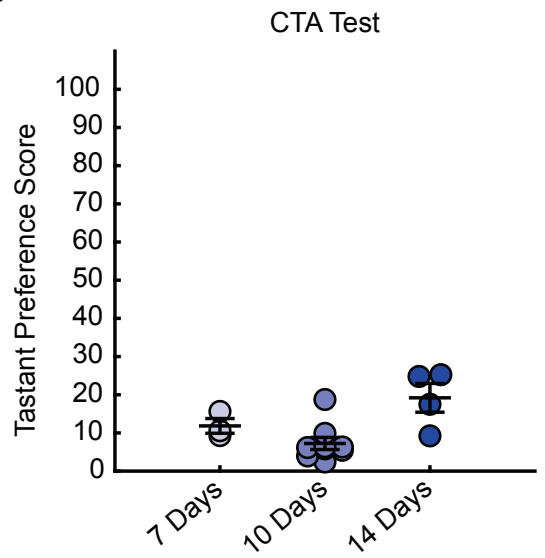


A

CTA

Virus Injection

$\bigvee 1$ Week

Conditioning

DOX ON

$24 \mathrm{Hrs} \nabla^{2} \mathrm{Hrs}$, 3Days

DOX OFF $\longdiv { \text { DOX ON } }$

B
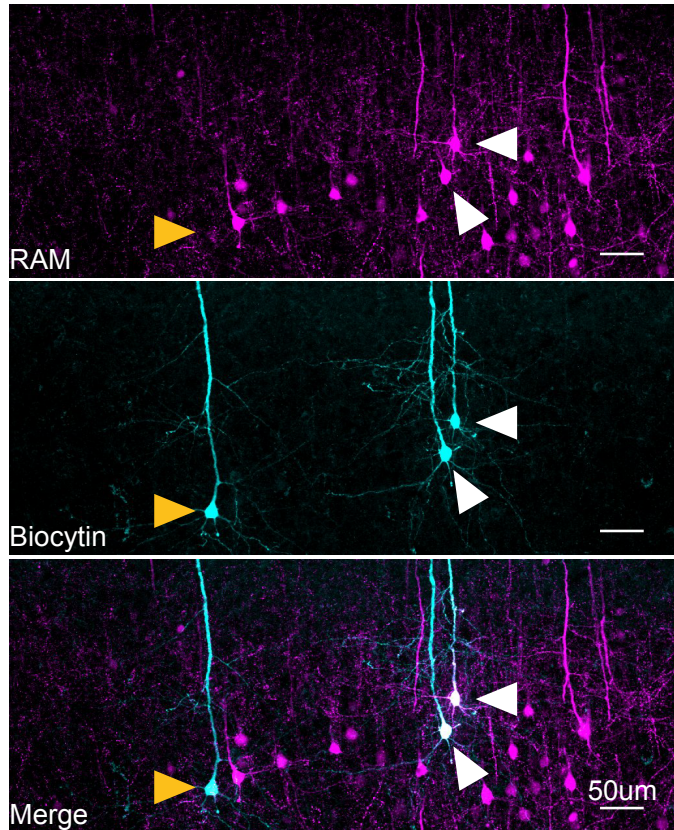

C

$\operatorname{MCTA}\left(\mathrm{RAM}^{+}\right)$

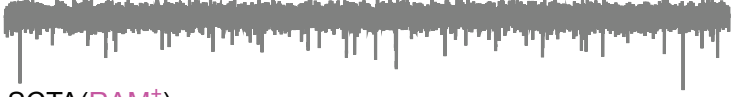

$\operatorname{SCTA}\left(\mathrm{RAM}^{+}\right)$

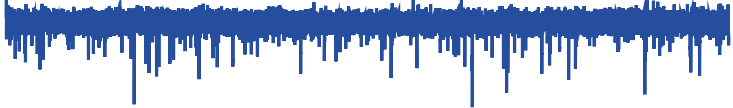
MCTA(RAM $\left.{ }^{-}\right)$

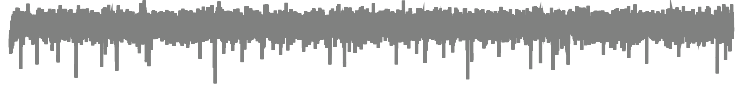
SCTA(RAM")

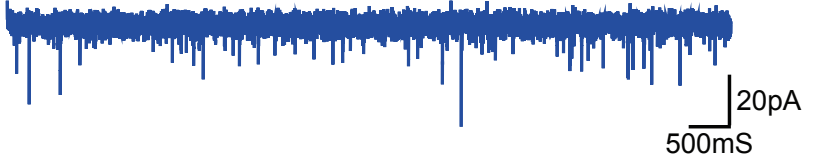

D

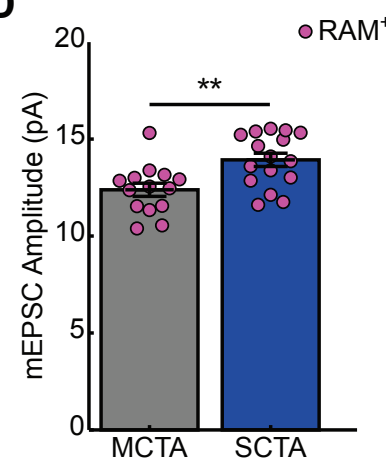

$E$

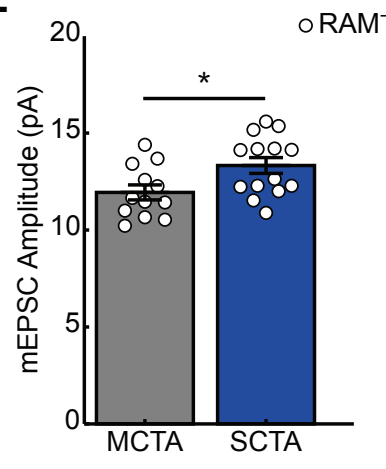




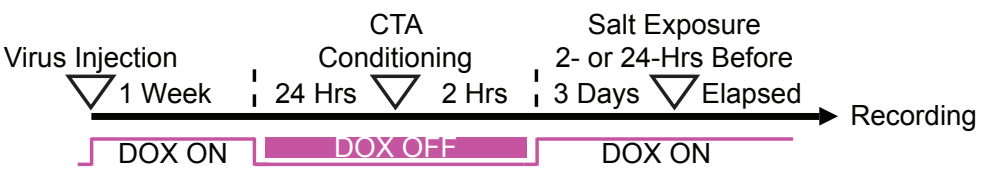

B

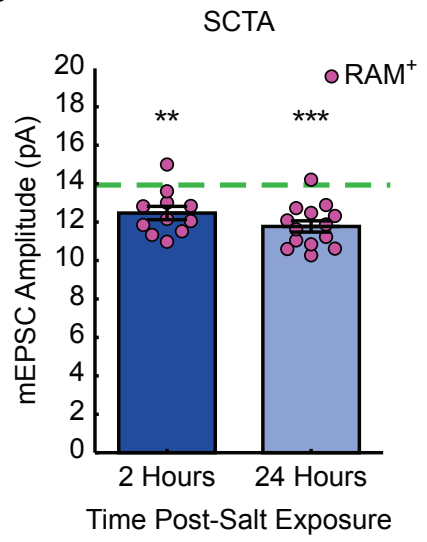

C

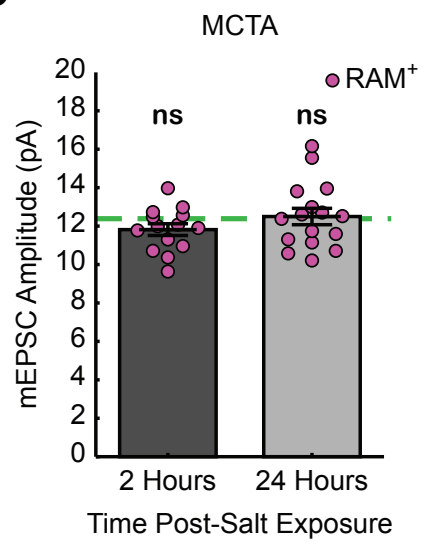


C

SCTA

Superficial Layers

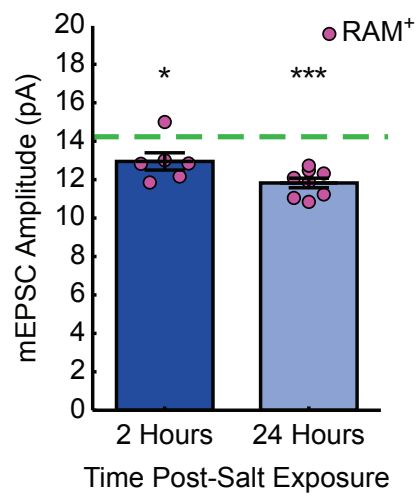

E

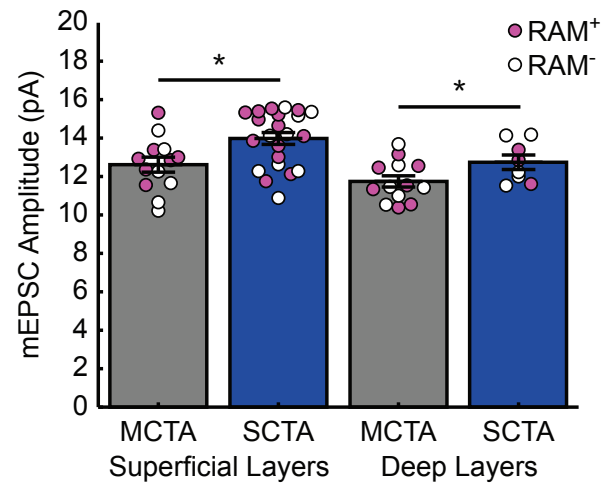

Layer Data - Pooled Cells

$\overline{100 \mathrm{um}}$

B Superficial Layers Deep Layers
E $\quad \begin{gathered}\text { MCTA } \\ \text { Superficial Layers }\end{gathered}$

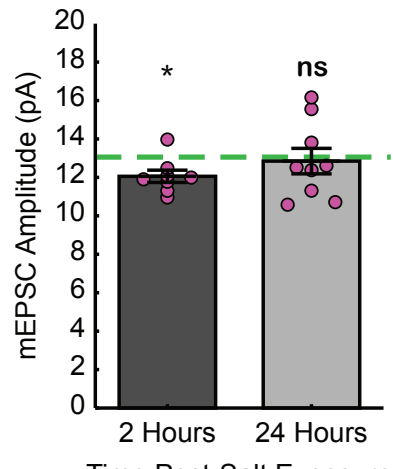

D

SCTA

Figure 5

Deep Layers

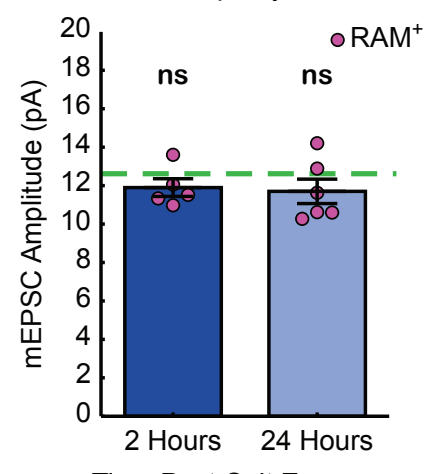

F

Time Post-Salt Exposure
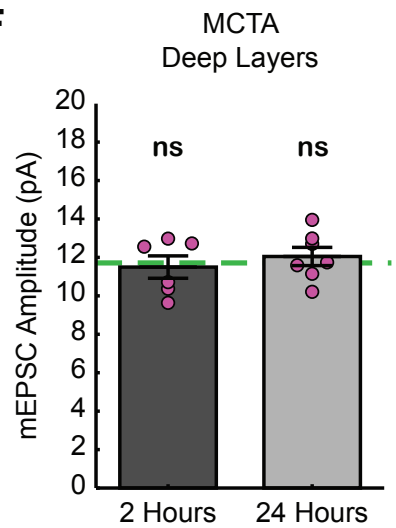

Time Post-Salt Exposure 